\title{
Metrological characterisation of rotating-coil magnetometer systems
}

\author{
Stefano Sorti ${ }^{1,2}$, Carlo Petrone ${ }^{2}$, Stephan Russenschuck ${ }^{2}$, Francesco Braghin ${ }^{1}$ \\ ${ }_{1}^{1}$ Politecnico di Milano, Department of Mechanical Engineering, Via La Masa, 1, 20156, Milano \\ 2 European Organization for Nuclear Research 1205 Geneva, Switzerland, 1211 Meyrin
}

\begin{abstract}
Rotating-coil magnetometers are among the most common and most accurate transducers for measuring the integral magnetic-field harmonics in accelerator magnets. The measurement uncertainty depends on the mechanical properties of the shafts, bearings, drive systems, and supports. Therefore, rotating coils require a careful analysis of the mechanical phenomena (static and dynamic) affecting the measurements, both in the design and in operation phases. The design phase involves the estimation of worst-case scenarios in terms of mechanical disturbances, while the operation phase reveals the actual mechanical characteristics of the system. In previous publications, we focused on modelling the rotating-coil mechanics for the design of novel devices. In this paper, we characterise a complete system in operation. First, the mechanical model is employed for estimating the forces arising during shaft rotation. Then, the effect of the estimated disturbances is evaluated in a simulated measurement. This measurement is then performed in the laboratory and the two results are compared. In order to characterise the robustness of the system against mechanical vibrations, different revolution speeds are evaluated. This work thus presents a complete procedure for characterising a rotating-coil magnetometer system.
\end{abstract}

\section{Section: RESEARCH PAPER}

Keywords: Magnetic measurements; magnetic field; rotating-coil magnetometers; rotating shaft

Citation: Stefano Sorti, Carlo Petrone, Stephan Russenschuck, Francesco Braghin, Metrological characterisation of rotating-coil magnetometer systems, Acta IMEKO, vol. 10, no. 2, article 6, June 2021, identifier: IMEKO-ACTA-10 (2021)-02-06

Section Editor: Giuseppe Caravello, Università degli Studi di Palermo, Italy

Received January 7, 2021; In final form April 13, 2021; Published June 2021

Copyright: This is an open-access article distributed under the terms of the Creative Commons Attribution 3.0 License, which permits unrestricted use, distribution, and reproduction in any medium, provided the original author and source are credited.

Corresponding author: Stefano Sorti, e-mail: stefano.sorti@polimi.it

\section{INTRODUCTION}

Rotating-coil systems are a special type of induction-coil magnetometers, based on Faraday's law of induction. They are used to measure integral field harmonics in the magnet bore. Rotating-coil systems consist of arrays of coils mounted on a rotating shaft, aligned with the magnet axis. The varying flux linkage with the coil induces a voltage. Typically, one long coil (or a chain of shorter coils) spans the entire magnet, including the fringe-field areas, because the transversal, integrated field is typically sufficient for beam tracking in particle accelerators [1].

Even if local measurements are required, through point-wise magnetometers [2], integral field is still a primary requirement.

Precise measurements of magnetic fields require a careful evaluation of the mechanical properties of the rotating coils, subject to static and dynamic forces [3]. This implies the need for the evaluation of vibrations [4]. Precautions for reducing the impact of mechanical deformations should also be taken at the design stage. A properly designed system should have natural frequencies higher than the operating frequencies [5].

Compensation schemes for the main field harmonic, commonly referred to as bucking, provide effective mitigation of spurious field harmonics caused by these vibrations [6] Nevertheless, the learned design methodologies are not always sufficient to match the requirements. Analytical formulas exist only for static phenomena such as misalignments and gravity [6]. Vibrations are instead modelled directly as spurious field harmonics in the measured field, and therefore they can only be evaluated in a prescribed field, as in [4]. The approach adopted in literature is typically to design the instrument aiming at a reasonably high stiffness, evaluating its compliance with controlled input like motor torque, as in [3]. Therefore, all the approaches proposed in the literature are limited in describing all the relevant mechanical phenomena, particularly shaft flexibility and propagation of mechanical vibration from motor and supports.

An analytical model for the mechanical description of rotating-coils was proposed in [7]. This model can predict the 


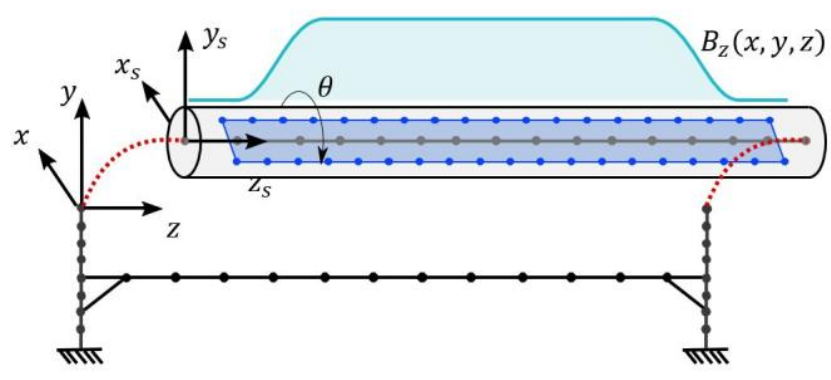

Figure 1. Layout of the mechanical model. The equations for the support structure and for the shaft are expressed in the fixed frame $(\boldsymbol{x}, \boldsymbol{y}, \boldsymbol{z})$ and in the rotating frame $\left(\boldsymbol{x}_{s}, \boldsymbol{y}_{s}, \boldsymbol{z}_{s}\right)$, respectively.

effect of static coil deformations, coil-axis to magnet alignment tolerances, and vibration modes on the measured field harmonics. The model was then expanded in a finite-element formulation (FEM) and applied to the design of a rotating-coil bench in [8]. This paper proposes a further refinement of the model, applied to the metrological characterisation of the designed rotating-coil system.

The shaft is an anisotropic rotor described in a non-inertial frame, thus including rotor-dynamics effects, coupled with steady space-frame support. Vibrations of the real system during operation are measured and introduced in simulations. A sample dipole magnet is measured both with the real system and through a simulation of the device in order to validate the robustness and the reliability of the system.

Therefore, the conclusion of this study is a complete magneto-mechanical description of a rotating-coil system in operation.

\section{THE MAGNETO-MECHANICAL MODEL}

The 3D finite-element method (FEM) [8] is adopted to describe rotating-coil shafts and their supporting structures. The two parts are modelled in different frames and coupled as described in the next section. The resulting mechanical deformation field of the shaft $\boldsymbol{u}(\boldsymbol{r})$ (where $\boldsymbol{u}$ is the displacement vector and $\boldsymbol{r}$ the position) is applied to the coil geometry to evaluate its effects on the magnetic measurements. The FEM model is shown in Figure 1.

\subsection{The mechanical model}

The model is based on Timoshenko beams with 12 degrees of freedom (DOF) per node, with the possibility of adding lumped masses, springs, and dampers. It is possible to include also non-ideal boundary conditions: clamped or hinged ends can be replaced by elastic foundations with a suitable stiffness.

The equations for the degrees of freedom (DOFs) $\boldsymbol{p}$ of the fixed support structure are:

$$
M \ddot{\boldsymbol{p}}+C \dot{\boldsymbol{p}}+K \boldsymbol{p}=\boldsymbol{f},
$$

where $M, K$ and $C$ are the mass, stiffness, and damping matrices of the system. Damping is characterised experimentally as modal damping [7], [8], and $\boldsymbol{f}$ accounts for external forces acting on the system. These include gravity, unbalancing, and vibration of moving parts.

The same equation holds for the rotating shaft but with the additional effects of not being in an inertial frame. The main advantage of adopting a rotating frame for the shaft is to have constant mechanical properties when reducing the shaft subsystem (shown below). This is a common approach for anisotropic shafts [9]. For the sake of simplicity, the rotating speed $\Omega$ is assumed to be constant, and therefore the angular displacement can be expressed as a function of time: $\theta=\Omega t$.

Let us consider the transformation expressing the rotation of the shaft $\boldsymbol{q}=R \boldsymbol{q}_{\mathrm{f}}$, where $R=R(\theta)$, and $\boldsymbol{q}_{\mathrm{f}}, \boldsymbol{q}$ are the shaft DOFs expressed in the fixed and rotating frames, respectively. Applying the transformation to the shaft equation in a fixed frame, following Eq. (1), and multiplying by $R$, the shaft equation in the rotating frame results is

$$
M \ddot{\boldsymbol{q}}+\left(C+2 R M \dot{R}^{\mathrm{T}}\right) \dot{\boldsymbol{q}}+\left(K-\Omega^{2} M\right) \boldsymbol{q}=R^{\mathrm{T}} \boldsymbol{f} .
$$

In this equation, the term added to $C$ denotes the Coriolis force, while the extra stiffness term is the centrifugal force.

Before coupling the subsystems, each one is reduced with the Craig-Bampton (CB) method [10]. The CB method is a popular technique that allows for an independent reduction of subdomains before the matrix assembly. It is based on splitting the set of DOFs of each subsystem into internal DOFs $\boldsymbol{q}_{\mathrm{i}}$ and boundary DOFs $\boldsymbol{q}_{\mathrm{b}}$ (or $\boldsymbol{p}_{\mathrm{i}}$ and $\boldsymbol{p}_{\mathrm{b}}$, respectively, for the support structure). Boundary DOFs are the ones at the interface between the subsystems and thus directly involved in the assembly of the full structure. The reduced set of DOF from the CB method is a combination of constraint modes $\Psi_{\mathrm{b}}$ and internal vibration modes $\Psi_{\mathrm{i}}$. They are expressed by the coordinate transformation

$$
\boldsymbol{q}=\left[\begin{array}{c}
\boldsymbol{q}_{\mathrm{b}} \\
\boldsymbol{q}_{\mathrm{i}}
\end{array}\right]=\left[\begin{array}{cc}
I & \mathbf{0} \\
\Psi_{\mathrm{i}} & \Psi_{\mathrm{b}}
\end{array}\right]\left[\begin{array}{l}
\boldsymbol{q}_{\mathrm{b}} \\
\boldsymbol{\varrho}_{\mathrm{i}}
\end{array}\right]
$$

where $I$ is the identity matrix, and $\boldsymbol{\varrho}_{\mathrm{i}}$ are modal coordinates of the shaft. The modal coordinates of supporting structure are denoted by $\boldsymbol{\pi}_{\mathrm{i}}$.

The computation of $\Psi_{\mathrm{i}}$ for the rotating shaft may be affected by the presence of non-symmetric matrices in the equation. To avoid a more complicated approach, typically involving left and right eigenvectors, it is proposed to compute $\Psi_{\mathrm{i}}$ as the free vibration modes of the shaft, neglecting the $\dot{\boldsymbol{q}}$ terms [9].

The computation of $\Psi_{\mathrm{b}}$ (for both subsystems) is performed by imposing fictitious unitary displacements to each boundary DOF and computing the static response:

$$
\Psi_{\mathrm{b}}=K_{\mathrm{i}, \mathrm{i}}{ }^{-1} K_{i, b},
$$

where $K_{\mathrm{i}, \mathrm{i}}$ and $K_{\mathrm{i}, \mathrm{b}}$ are the submatrices of the stiffness matrix $K$ accounting for internal-internal and internal-boundary interactions, respectively.

The reduction of each subsystem can be performed by truncating the $\Psi_{\mathrm{i}}$ set of modes. Different criteria can be enforced, mainly based on the maximum frequency component of the expected external inputs [11].

Finally, primal assembly [10] is adopted for the coupling between the support structure and rotating shaft. Only the subset $\boldsymbol{q}_{\mathrm{b}}$ must be transformed before coupling, resulting in $\boldsymbol{q}_{\mathrm{b}, \mathrm{f}}$. Modal coordinates $\boldsymbol{\varrho}_{\mathbf{i}}$ are not affected, as they are not in the physical space.

A generalised set of coordinates is introduced to account for both subsystems as

$$
\boldsymbol{u}=\underbrace{\left[\begin{array}{llll}
I & 0 & 0 & 0 \\
0 & I & I & 0 \\
0 & 0 & 0 & I
\end{array}\right]}_{L^{\mathrm{T}}}\left[\begin{array}{c}
\boldsymbol{\varrho}_{\mathrm{i}} \\
\boldsymbol{q}_{\mathrm{b}, \mathrm{f}} \\
\boldsymbol{p}_{\mathrm{b}} \\
\boldsymbol{\pi}_{\mathrm{i}}
\end{array}\right],
$$

where $I$ is the identity matrix and 0 is the zero matrix. 
The assembled equations finally result in

$$
\begin{aligned}
& L^{\mathrm{T}}\left[\begin{array}{cc}
M_{\mathrm{s}} & 0 \\
0 & M_{\mathrm{r}}
\end{array}\right] L \ddot{\boldsymbol{u}}+L^{\mathrm{T}}\left[\begin{array}{cc}
C_{\mathrm{s}} & 0 \\
0 & C_{\mathrm{r}}
\end{array}\right] L \dot{\boldsymbol{u}}+L^{\mathrm{T}}\left[\begin{array}{cc}
K_{\mathrm{s}} & 0 \\
0 & K_{\mathrm{r}}
\end{array}\right] L \boldsymbol{u} \\
& =L^{\mathrm{T}}\left[\begin{array}{l}
\boldsymbol{f}_{\mathrm{s}} \\
\boldsymbol{f}_{\mathrm{r}}
\end{array}\right],
\end{aligned}
$$

where the subscripts $\mathrm{s}$ and $\mathrm{r}$ identify the matrices for the support and the rotating shaft, respectively. Employing the FEM shape functions, the deformation field of the shaft can be computed and applied to the geometry of the coil.

\subsection{The magnetic model}

The mechanical model is coupled with the magnetic model by computing the magnetic flux linkage with the induction coil.

In order to calculate the system response to a given field distribution, the magnetic flux density is expressed analytically. The typical description for integral flux density is the 2D multipoles expansion [1]. However, for correct modelling, the full 3D field is required because long integral coils also intercept the magnet fringing field. Therefore, $3 \mathrm{D}$ pseudo-multipoles are adopted [12]:

$$
\begin{aligned}
& B_{r}(r, \varphi, z)=-\mu_{0} \sum_{n=1}^{\infty} r^{n-1}\left(\overline{\mathcal{C}}_{n}(r, z) \sin n \varphi\right. \\
& \left.+\overline{\mathcal{D}}_{n}(r, z) \cos n \varphi\right), \\
& B_{\varphi}(r, \varphi, z)=-\mu_{0} \sum_{n=1}^{\infty} n r^{n-1}\left(\tilde{\mathcal{C}}_{n}(r, z) \cos n \varphi\right. \\
& \left.-\widetilde{\mathcal{D}}_{n}(r, z) \sin n \varphi\right), \\
& B_{z}(r, \varphi, z)=-\mu_{0} \sum_{n=1}^{\infty} r^{n}\left(\frac{\partial \tilde{\mathcal{C}}_{n}(r, z)}{\partial z} \sin n \varphi\right. \\
& \left.+\frac{\partial \widetilde{\mathcal{D}}_{n}(r, z)}{\partial z} \cos n \varphi\right),
\end{aligned}
$$

where

$$
\begin{aligned}
& \overline{\mathcal{C}}_{n}(r, z)=n \mathcal{C}_{n, n}(z)-\frac{(n+2) \mathcal{C}_{n, n}^{(2)}(z)}{4(n+1)} r^{2}+\cdots \\
& \tilde{\mathcal{C}}_{n}(r, z)=\mathcal{C}_{n, n}(z)-\frac{\mathcal{C}_{n, n}^{(2)}(z)}{4(n+1)} r^{2}+\cdots
\end{aligned}
$$

In the interest of brevity, the similar expressions for the skew components $\widetilde{\mathcal{D}}_{n}, \overline{\mathcal{D}}_{n}$ have been omitted here. It is, therefore, possible to compute the magnetic flux density at any point in space. The flux linkage in the coils is then calculated numerically with

$$
\Phi=\int_{\mathcal{A}} \mathbf{B} \cdot \mathrm{d} \mathbf{a} .
$$

In a discrete setting, the flux increments $\phi_{m}$ for any angular positions $\theta_{m}$ can be developed into a discrete Fourier series:

$$
\Psi_{n}=\sum_{m=0}^{M-1} \phi_{m} \cdot e^{\frac{-i 2 \pi m n}{N}} .
$$

This yields the harmonic content of the response:

$$
C_{n}\left(r_{0}\right)=r_{0}^{n-1} \frac{\Psi_{n}}{k_{n}}
$$
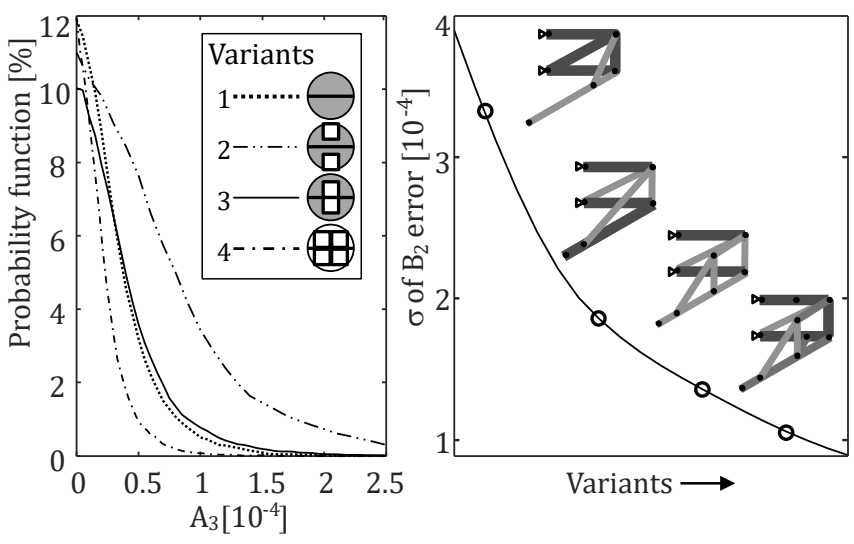

Figure 2. Overview of the design phase for the shaft, on the left, and for the support, on the right (adapted from [8]).

where $C_{n}^{\mathrm{a}}\left(r_{0}\right)=B_{n}^{\mathrm{a}}\left(r_{0}\right)+i A_{n}^{\mathrm{a}}\left(r_{0}\right)$ are the measured (apparent) field harmonics and $k_{n}$ are the coil sensitivity factors for the $n$ th field harmonic:

$$
k_{n}=\frac{N_{T} L_{c}}{n}\left(r_{2}^{n}-r_{1}^{n}\right),
$$

where $N_{T}$ is the number of coil turns, $L_{c}$ the total length of the coil and the two radii are the position of the go and return wires of the coil. The $k_{n}$ express the integral sensitivity of the coil and are therefore not functions of $z$. The differences between the imposed $A_{n}, B_{n}$ and the apparent $A_{n}^{\mathrm{a}}, B_{n}^{\mathrm{a}}$ coefficients at the reference radius of measurement are the main figure of merit to evaluate the effects of mechanical defects.

\section{THE MEASUREMENT SYSTEM REVIEW}

The proposed model was used to design the measurement system, as described in [8]. A brief recap of the design considerations is given before presenting the model of the final system.

\subsection{The design process}

The design process was based on separate studies of the two subsystems. The shaft was designed considering an approximated (non-optimal) support. Then the supporting structure was designed for the expected worst-case scenario (i.e. for the least stiff shaft expected to be mounted on the support). A recall of the design procedure is presented in Figure 2. On the left, the probability density function of errors in the field multipole $A_{3}$ for different design iterations of the shaft, from bulk resin (1) to carbon-only shaft (4). On the right, the standard deviation of the error in measuring the main component of a quadrupole $B_{2}$ for different designs of the supporting structure.

The shaft was designed for measuring the field harmonics in dipoles and quadrupole magnets. Compensation schemes were applied in simulating the measurement procedure. In order to calculate the performance of the compensation in the design, coil imperfections were introduced as Gaussian distributions. The external forces considered were gravity, support vibrations (in the form of harmonic forces on the bearings), bearing-frictionrelated torque, and shaft unbalancing due to eccentricity and sag. The boundary conditions for the shaft were elastic supports (lumped springs and dampers), accounting for the equivalent stiffness of the supporting structure. 

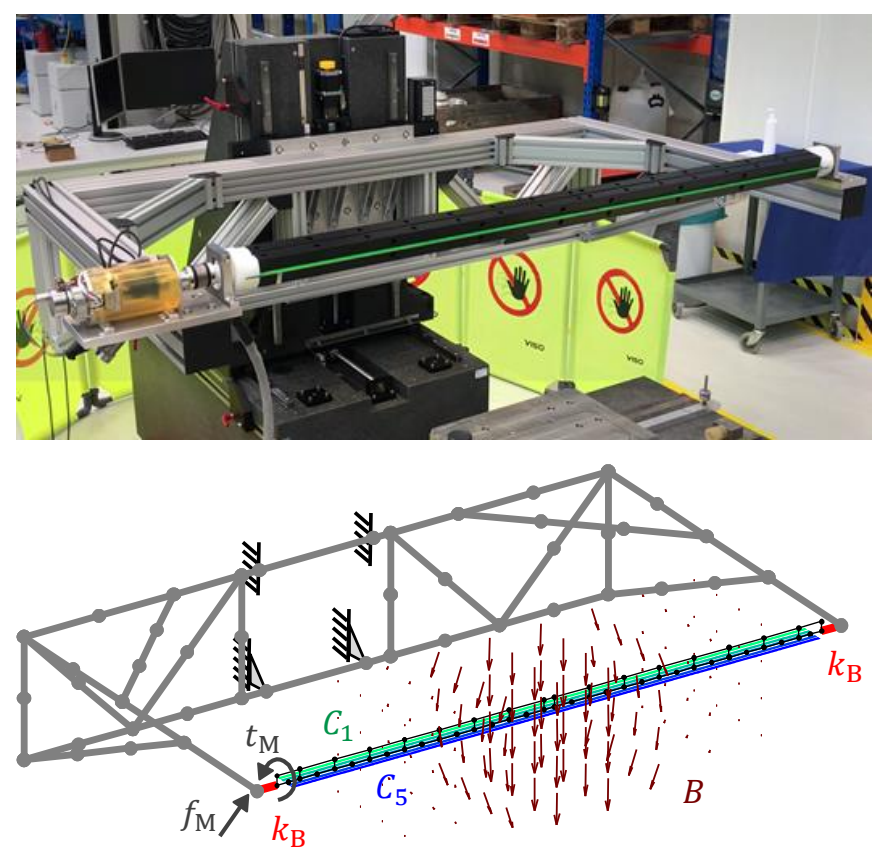

Figure 3. The rotating-coil system without the magnet (top) and its magnetomechanical model (bottom). A few elements are highlighted: the force $\boldsymbol{f}_{M}$ and the torque $\boldsymbol{t}_{\mathbf{M}}$ generated by the motor, the bearing stiffness matrix $\boldsymbol{k}_{\mathrm{B}}$, the array of coils $\left(\boldsymbol{c}_{\mathbf{1}}\right.$ to $\boldsymbol{c}_{\mathbf{5}}$ ) and the magnetic flux density $\boldsymbol{B}$ to be measured. The rigid bodies accounting for motor and shaft ends are omitted.

Regarding the design of the support, the error of the main field component was the most relevant figure of merit. The design process was thus an iteration of different space-frame layouts for the support structure.

\subsection{The model of the measurement system}

The complete model of the measurement system is shown in Figure 3 (top image). It consists of a 1.5-m-long shaft made of carbon-fiber composite profiles, supporting a printed circuit board (PCB) coil array. The array is made of five equal radial coils of $1.48 \mathrm{~m}$ length, $11.2 \mathrm{~mm}$ width, and 60 turns. The diameter of the shaft is $72 \mathrm{~mm}$. The supporting structure is a two-meter-wide aluminium frame. The boundary conditions for the support structure are two clamped and two pinned nodes. This comes from the different layouts of the joints and aims at being conservative in terms of mechanical stiffness.

Figure 3 (bottom image) also shows the modelled magnetic field for the characterisation of the system, which is the main topic of the next section. The shaft model consists of two parallel beams, representing the $\mathrm{PCB}$ and the carbon profiles, respectively. Bolt joints are modelled as rigid links between mesh nodes. Bearing stiffness is included in the interface between the shaft and the structure as a set of lumped linear springs in the fixed frame. The flexible joint, which links the shaft with the motor, is included in the bearing stiffness matrix as a torsional spring. The motor-drive unit is introduced as a rigid body on one end of the structure. Forces and torques generated by the rotation are modelled as external loads.

The support structure weights $65 \mathrm{~kg}$, it guarantees a stiffness at the tip of a minimum $1.5 \times 10^{5} \mathrm{~N} / \mathrm{m}$ in all three directions and has its first natural frequency at $28 \mathrm{~Hz}$. The shaft is approximately $2.5 \mathrm{~kg}$ and has the first natural frequency (a bending mode) at $74 \mathrm{~Hz}$.

\subsection{The expanded model for input estimation}

The effects of the motor on the mechanical system are modelled as equivalent lumped inputs. In particular, we introduce a force $\boldsymbol{f}_{\mathrm{M}}$ (with unknown magnitude and direction) and a torque $t_{\mathrm{M}}$ about the shaft rotation axis. The force and torque are applied in the node supporting the shaft on the motor side. Due to the linking between the structure and the shaft (through stiffness $k_{\mathrm{B}}$ ), their effects are visible on the coils and therefore have an effect on the magnetic measurements.

Due to the simplification in the modelling of the motor effects, it is necessary to provide the model with the vector of forces and torque $\boldsymbol{g}=\left[\boldsymbol{f}_{\mathrm{M}}, t_{\mathrm{M}}\right]$.

In order to minimise the impact of the mechanical measurements on the magnetic measurements, an indirect measurement of $\boldsymbol{g}$ is performed. Instead of mounting force sensors at the joints between the parts, like torque transducers [14], accelerometers are mounted at the shaft supports. Therefore, accelerations are measured, and forces are estimated. To perform this operation, a Kalman Filter is introduced.

The mechanical model of Eq. (6) is expanded to include the inputs in Kalman Filter's estimation [13]. It is required to write the model in state-space form, collecting the variables in the vector $\boldsymbol{x}=[\dot{\boldsymbol{u}}, \boldsymbol{u}]$. Before assembling the estimator, it is suggested to convert the dynamic system in a discrete-time domain (with time step $\Delta \mathrm{t}$ ). It is, therefore, possible to write:

$$
\left[\begin{array}{l}
\boldsymbol{x}_{k+1} \\
\boldsymbol{g}_{k+1}
\end{array}\right]=\left[\begin{array}{cc}
A & B \\
0 & I
\end{array}\right]\left[\begin{array}{l}
\boldsymbol{x}_{k} \\
\boldsymbol{g}_{k}
\end{array}\right]+\left[\begin{array}{cc}
L & 0 \\
0 & \Delta \mathrm{t} I
\end{array}\right]\left[\begin{array}{l}
\boldsymbol{Z} \\
\boldsymbol{w}
\end{array}\right]
$$

In Eq. (16), the first term stems from Eq. (6), while the second term is added for the sake of state estimation. It contains the mechanical disturbances $L \boldsymbol{Z}$ and the fictitious disturbances for the input $\Delta \mathrm{t} I \boldsymbol{w}$. This term is required to consider time-varying inputs in the estimation, otherwise, the inputs would obey $\boldsymbol{g}_{k+1}=\boldsymbol{g}_{k}$. More specifically, $L$ can be assumed to be the identity matrix in case no external disturbances are expected, while $\boldsymbol{Z}$ and $\boldsymbol{w}$ are zero-mean, normally-distributed disturbances with covariance matrices $Q_{z}=\operatorname{cov}(\boldsymbol{z}, \boldsymbol{z})$ and $Q_{w}=\operatorname{cov}(\boldsymbol{w}, \boldsymbol{w})$. The Kalman filter also requires an output equation, which is in our case the subset from Eq. (6) accounting for the accelerations of the nodes at which position the accelerometers are mounted. The output is expected to suffer from measurement noise, assumed to be a zero-mean, normally-distributed variable $\boldsymbol{n}$ with covariance matrix $R=\operatorname{cov}(\boldsymbol{n}, \boldsymbol{n})$.

\section{EXPERIMENTAL VALIDATION}

The experimental validation campaign aims at two objectives: to evaluate the accuracy of the model in predicting the outcome of a magnetic measurement, and to characterise metrologically the effect of vibrations in the real system.

Regarding the model-prediction capabilities, the model is the same as the one in the design phase. It is based on conservative hypotheses for boundary condition of the structure (Figure 3 and Section 3.2) and without updates based on vibration measurements. The comparison between the model and the measurements is therefore expected to be consistent up to a safety factor. Approaches to update the system model in order to replicate the real system more accurately are discussed in the next section. 


\subsection{Experimental setup}

As a first case study, a dipole magnet is selected. It is a $0.4 \mathrm{~m}$ long dipole from the CERN East Area, with a nominal integral field of $0.36 \mathrm{Tm}$ at $240 \mathrm{~A}$ [15]. Future studies will also cover the case of quadrupoles and higher-order magnets.

The magnetic flux density distribution generated by the magnet was already measured with a short rotating-coil, and pseudo-multipoles were computed up to the $5^{\text {th }}$ component. Pseudo-multipoles coefficients are therefore available for the measurement model.

The magnetic measurement performed with the system under investigation is a rotating-coil measurement assessing both the integral absolute field and the integral multipole coefficients. Therefore, the fluxes from the main coil $c_{1}$ and from the compensation scheme $c_{1}-c_{3}$ are acquired

The $k_{1}$ sensitivity factor for each of the coil of the array is calibrated in a reference magnet. In order to match the calibrated area, coil widths in the model are adjusted. This is also to decrease the performance of bucking compensation in the model, aiming at more realistic values. Coefficients $k_{n}$ for $n>1$ are computed directly from the nominal geometry of the model.

A single measurement consists in a revolution at constant speed. Different speeds are considered for both the mechanical and the magnetic measurement campaigns so that, adopting the units of revolutions-per-minute, $\Omega \in[30,45,60,75,90,105,120]$. In order to prevent unpredicted phenomena (like thermal transients) from correlating with the speed wrongly, the different values for $\Omega$ are evaluated in a randomised order. 20 repetitions are performed per each speed.

For the mechanical evaluation of the measuring system, a Leica geosystem is employed for the alignment with respect to the magnet. In addition, two tri-axial accelerometers $(6 \mathrm{~g}$ range, 0-200 Hz bandwidth) are mounted on the support structure next to the coil bearings to assess the vibrations of the system. Given their weight of $50 \mathrm{~g}$, they have been neglected in the mechanical model.

Due to the low values expected for accelerations, the accelerometers have been calibrated [16] in the range of $\pm 0.5 \mathrm{~g}$ (or $0.5 \mathrm{~g}-1.5 \mathrm{~g}$ for the axes parallel to gravity): each device was mounted on a support that allows a controlled angular displacement with respect to gravity (accuracy $0.02 \mathrm{mrad}$ ). DC acceleration is then measured in a series of known angular positions, so that the gravity component per each axis could be computed. The overall accuracy is estimated to be $0.0025 \mathrm{~m} / \mathrm{s}^{2}$. This value includes the uncertainty due to the sensor noise, averaged over 20 repetitions.

The impact of accelerometer accuracy on the force estimation is estimated to be about $0.3 \%$ in amplitude; it has thus negligible effects on the measurement. The main source of uncertainty for the estimation is the model itself, which is in fact, the focus of the validation procedure.

\subsection{Mechanical measurements results}

The most relevant figure of merit for the mechanical alignment of the system is the misalignment between magnet axis and coil rotation axis. After the alignment procedure, the final angle between the two axes resulted in $0.052 \mathrm{mrad}$.

The positioning error is estimated to be less than $20 \mu \mathrm{m}$, which is the overall accuracy of the geosystem, while the linear stages can perform smaller motions relying on a sub-micrometerresolution encoder.
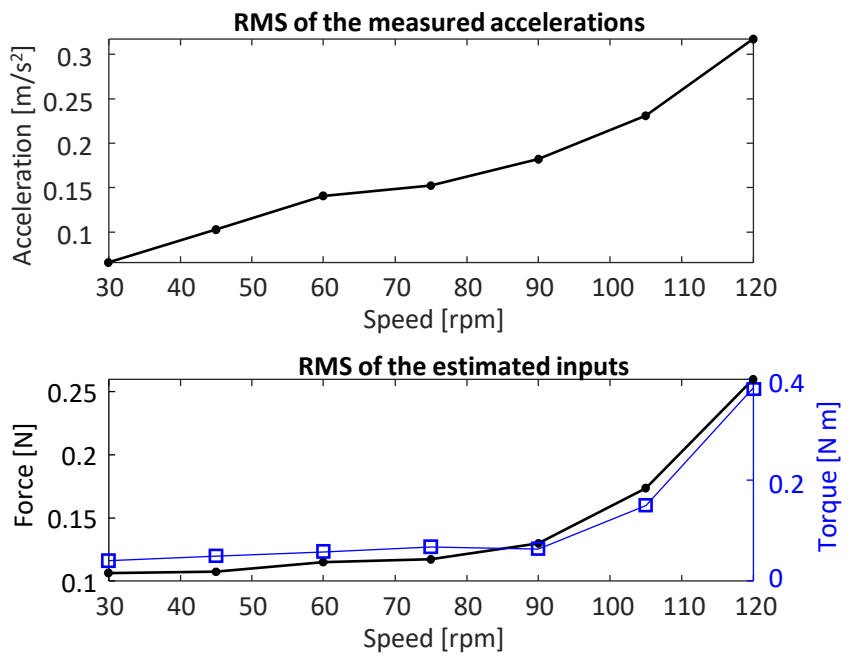

Figure 4. RMS values for the measured accelerations (averaging all the accelerometers) and for the estimated force magnitude and torque. The RMS are computed over the full bandwidth $(0-200 \mathrm{~Hz})$ of the accelerometers.

However, these positioning errors are negligible for the proposed magnetic measurements (estimated to result in errors of the order of $\left.10^{-8}\right)$.

The vibrations are measured for different rotation speeds of the shaft. For each of the speeds considered, the estimation procedure is performed on a series of 20 revolutions.

Figure 4 shows the RMS of the measured vibrations and the estimated force and torque as the rotating speed function.

\subsection{Magnetic measurements results}

The main figure of merit for the performance of the measurement system is the result of the magnetic measurement. Consistent with the mechanical measurements, different values are considered for the rotation speed of the shaft. The twofold scope of the campaign is to validate the robustness of the measurement results against the mechanical vibrations and the capability of the model in reproducing the system behaviour.

Figure 5 shows the measurements of the absolute integral field as a function of the speed. Each rotation is processed independently to evaluate the dispersion caused by random
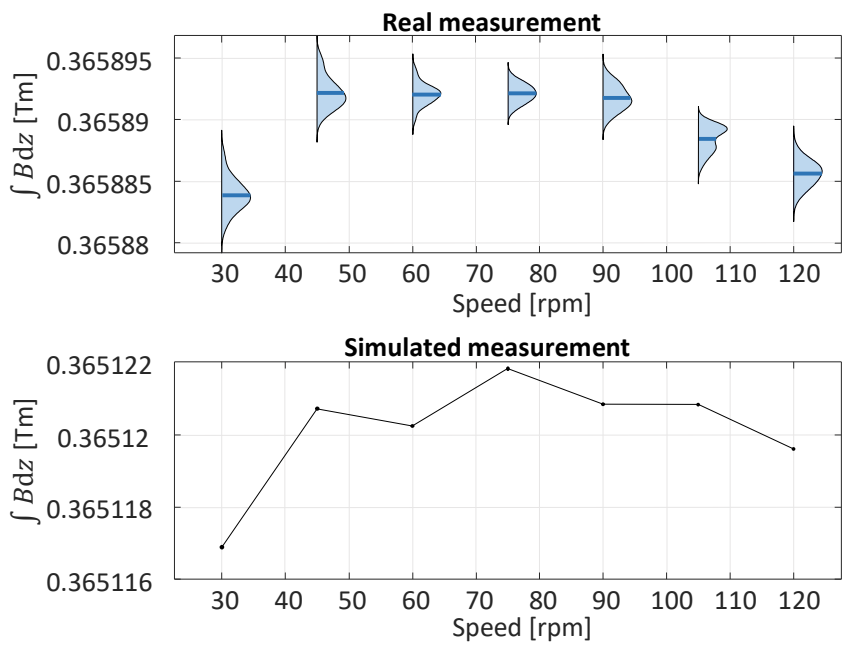

Figure 5. Integral absolute field resulting from measurements (top) and simulations (bottom). The measured results are presented by the distribution of 20 rotations (blue line is the average). 
disturbances and noise. The distribution shown is a probability density estimate based on a normal kernel function.

The overall spread concerning all the measured values is 0.4 units $\left(10^{-4}\right.$ of the average field), while averaging the turns, the spread is 0.2 units. Moreover, the trend of the mean values as a function of the speed is not monotonic, as is the RMS of the accelerations. The reason is that other phenomena are speedrelated such as the signal-to-noise ratio for the acquisition system. Therefore, it is plausible to identify a relative accuracy of \pm 0.1 units from the mechanical vibrations on the absolute integral field.

The second plot in Figure 5 shows the simulated measurement of the integral field. The difference between the measured and simulated values is on average 21 units, while the spread between measurements is 0.15 units. Comparing the trend, these results confirm that the model correctly predicted the amount of disturbances from mechanical vibration in the magnetic measurement.

The error in the absolute field can be explained by the missing high-order harmonics in the simulation. As the absolute field is constant with respect to vibration amplitude, this is not investigated further.

Figure 6 shows the measurement results of the integral multipoles. The multipoles coefficients are measured with both the main coil $c_{1}$ and the compensation scheme $c_{1}-c_{3}$. It is therefore possible to appreciate the performance enhancement of the compensation in the measurement of high-order multipoles (between $10^{\text {th }}$ and $15^{\text {th }}$ ). Nevertheless, the effects of vibrations on the multipoles measured by the main coil are at most 0.1 units for the speeds considered. Thus, the rotating-coil system yields measurement results better than one unit, even without a compensation scheme. As far as the simulated measurement is concerned, the multipoles from the main coil are given. Thus, it is possible to appreciate the matching of the behaviour for both the multipoles values and their trends as a
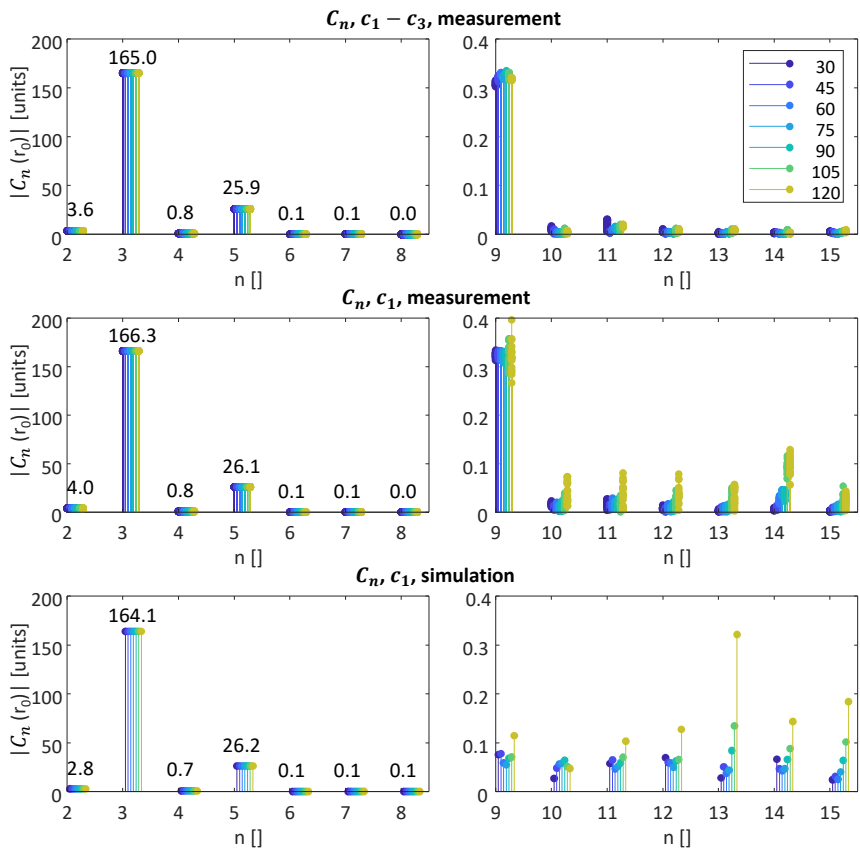

Figure 6. Field multipoles (modulus) as measured with the compensated scheme $\boldsymbol{c}_{\boldsymbol{1}}-\boldsymbol{c}_{\mathbf{3}}$ (top), by the main coil $\boldsymbol{c}_{\mathbf{1}}$ (middle), and as simulated for $\boldsymbol{c}_{\boldsymbol{1}}$ (bottom). For the measured quantities, each rotation is processed independently and pictured by a dot. function of the speed. In particular, the model is slightly overestimating the effect of the mechanical vibrations on highorder multipoles, but this is consistent with the model's expected behaviour. The field harmonic of order 9 was not included in the pseudo-multipoles provided to the model.

\section{CONCLUSIONS}

The proposed magneto-mechanical model, already applied to the design of a rotating-coil system, is now validated against the actual device in a real magnetic measurement campaign. The vibrations of the system during the measurement have been recorded, and the corresponding input was estimated. The 3D magnetic flux density of the magnet was provided, and the measurement procedure was simulated.

The results agree with the trends and the magnitudes of the real measurement. If a closer matching is required, the model should be updated accordingly. This updating would require a proper mechanical measurement campaign to experimentally characterise all the system features (for instance, by modal analysis, with an array of accelerometers and a controlled input). Nevertheless, the real system magnetic measurements confirmed that the performance required in the design phase had been exceeded, with an overall error by mechanical vibrations estimated to a few units of $10^{-5}$.

Therefore, we can conclude that the proposed procedures have led to the design, construction, and commissioning of a novel rotating-coil magnetometer system with unprecedented control over some mechanical properties typically critical for the magnetic measurement result.

\section{REFERENCES}

[1] S. Russenschuck, Field computation for accelerator magnets, WileyVCH, 2010.

DOI: $\underline{10.1002 / 9783527635467}$

[2] D. Popovic Renella, S. Spasic, S. Dimitrijevic, M. Blagojevic, R. S Popovic, An overview of commercially available teslameters for applications in modern science and industry, Acta IMEKO 6(1) (2017), pp. 43-49.

DOI: $10.21014 /$ acta imeko.v6i1.312

[3] J. Billan, J. Buckley, R. Saban, P. Sievers, L. Walckiers, Design and test of the benches for the magnetic measurement of the LHC dipoles, IEEE Transactions on Magnetics 30(4) (1994), pp. 26582661.

DOI: $10.1109 / 20.305826$

[4] N. R. Brooks, L. Bottura, J. G. Perez, O. Dunkel, L. Walckiers, estimation of mechanical vibrations of the LHC fast magnetic measurement system, IEEE Transactions on Applied Superconductivity 18(2) (2008), pp. 1617-1620.

DOI: $10.1109 /$ TASC.2008.921296

[5] G. Tosin, J. F. Citadini, E. Conforti, Long rotating coil system based on stretched tungsten wires for insertion device characterization, IEEE Transactions on Instrumentation and Measurement 57(10) (2008), pp. 2339-2347. DOI: $10.1109 /$ TIM.2008.922093

[6] L. Walckiers, Magnetic measurement with coils and wires, CERN Accelerator School: Magnets, Bruges, Belgium 16-25 June 2009. Online [Accessed 14 June 2021]

https://cds.cern.ch/record/1345967

[7] S. Sorti, C.Petrone, S. Russenschuck, F. Braghin, A magnetomechanical model for rotating coil magnetometers, Nuclear Instruments and Methods in Physics Research Section A, 984 (2020) art. 164599.

DOI: $10.1016 /$ i.nima.2020.164599

[8] S. Sorti, C.Petrone, S. Russenschuck, F. Braghin, A mechanical analysis of rotating-coil magnetometers, IMEKO TC4 2020, 
virtual conference, Palermo, Italy, 14-16 September 2020. Online [Accessed 14 June 2021]

https://www.imeko.org/publications/tc4-2020/IMEKO-TC4 2020-38.pdf

[9] G. Genta, Dynamics of Rotating Systems, Springer, 2005 edition. DOI: $10.1007 / 0-387-28687-X$

[10] Mapa, Lidianne de Paula Pinto, Francisco de Assis das Neves, Gustavo Paulinelli Guimarães. Dynamic substructuring by the Craig-Bampton method applied to frames, Journal of Vibration Engineering \& Technologies 9(2021), pp. 257-266.

DOI: $10.1007 /$ s42417-020-00223-4

[11] B. Besselink, et al. A comparison of model reduction techniques from structural dynamics, numerical mathematics and systems and control, Journal of Sound and Vibration 332(19) (2013), pp. 4403 4422.

DOI: $10.1016 /$ i.jsv.2013.03.025

[12] S. Russenschuck, G. Caiafa, L. Fiscarelli, M. Liebsch, C. Petrone, P. Rogacki, Challenges In Extracting Pseudo-Multipoles From Magnetic Measurements, 13 th Int. Computational Accelerator Physics Conf. ICAP2018, Key West, FL, USA, 20-24 October
2018, 6 pp

10.18429/JACoW-ICAP2018-SUPAG03

[13] J. C. Berg, A. K. Miller, Force estimation via Kalman filtering for wind turbine blade control, Structural Dynamics and Renewable Energy, Volume 1. Springer, New York, NY, 2011, pp. 135-143. DOI: $10.1007 / 978-1-4419-9716-613$

[14] R. S. Oliveira, R. R. Machado, H. Lepikson, T. Fröhlich, R. Theska, A method for the dynamic calibration of torque transducers using angular speed steps, Acta IMEKO 8(1) (2019), pp. 13-18.

DOI: $10.21014 /$ acta imeko.v8i1.654

[15] R. Lopez, J. R. Anglada, The New Magnet System for the East Area at CERN, IEEE Transactions on Applied Superconductivity 30(4) (2020), pp. 1-5.

DOI: $10.1109 /$ TASC. 2020.2972834

[16] G. D’Emilia, A. Gaspari, E. Natale, Dynamic calibration uncertainty of three-axis low frequency accelerometers, Acta IMEKO 4(4) (2015), pp. 75-81.

DOI: $10.21014 /$ acta imeko.v4i4.239 